\title{
Diffractive beam splitter characterization via a power-recycled interferometer
}

\author{
Daniel Friedrich, ${ }^{1, *}$ Oliver Burmeister, ${ }^{1}$ Alexander Bunkowski, ${ }^{1}$ Tina Clausnitzer, ${ }^{2}$ Stephan Fahr, ${ }^{2}$ \\ Ernst-Bernhard Kley, ${ }^{2}$ Andreas Tünnermann, ${ }^{2}$ Karsten Danzmann, ${ }^{1}$ and Roman Schnabel ${ }^{1,3}$ \\ ${ }^{1}$ Albert-Einstein-Institut Hannover, Max-Planck-Institut für Gravitationsphysik und Institut für Gravitationsphysik der \\ Leibniz Universität Hannover, Callinstrasse 38, 30167 Hannover, Germany \\ ${ }^{2}$ Institut für Angewandte Physik, Friedrich-Schiller-Universität Jena, Max-Wien-Platz 1, 07743 Jena, Germany \\ ${ }^{3}$ roman.schnabel@aei.mpg.de \\ *Corresponding author: daniel.friedrich@aei.mpg.de
}

Received September 18, 2007; revised November 5, 2007; accepted November 14, 2007; posted November 19, 2007 (Doc. ID 87652); published January 4, 2008

We used the high-precision laser interferometer technique of power recycling to characterize the optical loss of an all-reflective grating beam splitter. This beam splitter was used to set up a Michelson interferometer with a power-recycling resonator with a finesse of 883. Analyzing the results obtained, we determined the beam splitter's total optical loss to be $(0.193 \pm 0.019) \%$. Low loss all-reflective beam splitters might find application in future high-power laser interferometers for the detection of gravitational waves. () 2008 Optical Society of America

OCIS codes: $050.1950,120.3180,230.1360$

Reflection gratings are being investigated as alternatives to conventional transmissive beam splitters in high-power laser interferometers [1-3]. Although residual absorption of high quality optics can be as small as 0.25 parts in $10^{6}$ (ppm)/cm [4], this loss still leads to thermal effects inside an optical substrate when operated at high optical powers. Thermal deformations and thermal lensing limit the storable power inside an interferometer and hence constrain the shot-noise-limited sensitivity [5]. Current interferometric gravitational wave detectors are advanced Michelson interferometers that use the technique of power recycling (PR) [6]. In this technique an optical cavity is built around the interferometer's central beam splitter to achieve a laser power build-up. Light power in excess of $1 \mathrm{~kW}$ at the beam splitter has already been achieved [4]. To obtain a high PR-build-up factor a high interference contrast at the beam splitter and low optical loss of the beam splitter are mandatory. The natural question is, can these requirements be met by all-reflective grating beam splitters.

In this Letter we present the experimental realization of a Michelson interferometer with an allreflective grating beam splitter and PR. We realized a high interference contrast, although in such a topology the laser beam shape is necessarily different in both interferometer arms. The PR cavity was utilized as a new tool to precisely measure the total optical loss of the diffractive beam splitter for monochromatic light of a given wavelength.

Figure 1 illustrates the equivalence of the optical functionality of a conventional beam splitter and an all-reflective beam splitter with two diffraction orders within a Michelson interferometer. The grating investigated here was designed as a 50/50 beam splitter for an $s$-polarized laser beam at $\lambda=1064 \mathrm{~nm}$ having its zeroth and first diffraction orders nearly $90^{\circ}$ apart from each other [7]. Note that for a grating period $d$ and angle of incidence $\phi_{\text {in }}$ the angle of the $m$ th order $\phi_{m}$ is given by the grating equation $\sin \left(\phi_{\text {in }}\right)+\sin \left(\phi_{m}\right)=m \lambda / d$. For both Michelson interferometers in Fig. 1 the antisymmetric output port points back toward the laser. This port contains the laser beams being twice reflected, transmitted, or diffracted. The signal port contains the output beams having a symmetric optical path of either one reflection and transmission (rt, tr) or one reflection and diffraction $(01,10)$. If the arm lengths of the interferometer are controlled to provide a dark signal port, all the laser power is back reflected toward the laser and can be "recycled" by a mirror $\mathrm{M}_{\mathrm{PR}}$ establishing a (high-finesse) PR cavity.

However, the two setups in Fig. 1 also show distinct differences. For a conventional beam splitter the reflected and transmitted beams have the same beam profile and propagate in a common manner, if the astigmatism caused by the transmission through the substrate is compensated by means of an additional substrate in the path of the reflected light. If the two interferometer arms are identical in terms of lengths, optical loss, and the end mirrors' radii of curvature, the interference contrast of the beams coming from the interferometer's arms should be perfect and in particular should not depend on the geometry of the incident beam. For a grating beam splitter the ze-

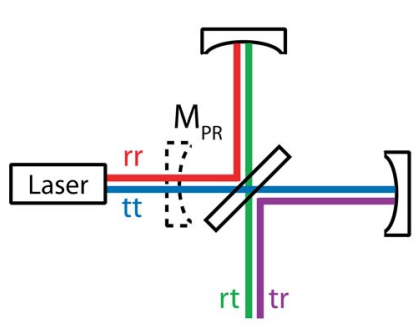

(a)

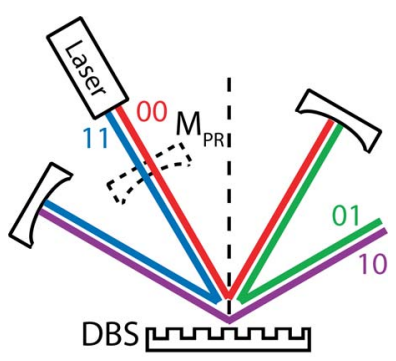

(b)
Fig. 1. (Color online) Comparison of a (a) conventional with an all-reflective Michelson interferometer with a (b) diffractive beam splitter (DBS). The additional mirror $\mathrm{M}_{\mathrm{PR}}$ can establish a PR cavity to increase the light power inside the interferometer. 
roth and first orders have different deflection angles, resulting in different beam profiles in the interferometer arms. An incident beam of circular shape remains circular in its zeroth diffraction order but is elliptical in its first order [8] with a changed beam diameter parallel to the diffraction plane.

To achieve a high interference contrast for an interferometer with identical arms the incident beam must also be taken into account. Figure 2 shows that for a certain $\mathrm{TEM}_{00}$ input mode (negative $z$ values), two spherical end mirrors at the same distance from the grating beam splitter can provide perfect mode overlap at the antisymmetric interferometer output port. Most importantly, the mode produced also matches the input mode, which is a requirement for the PR technique to preserve the interference contrast. The curves further illustrate how the beam widths parallel to the diffraction plane evolve during propagation in the interferometer arms. Here we used the $A B C D$ matrix formalism for Gaussian beams [8]. To realize the PR technique in this optical configuration we found the following condition for the distance $l_{\mathrm{PR}}$ between the $\mathrm{PR}$ mirror $\mathrm{M}_{\mathrm{PR}}$ and the grating beam splitter:

$$
l_{\mathrm{PR}}=\left|z_{0}+R_{\mathrm{PR}} / 2-\sqrt{R_{\mathrm{PR}}^{2} / 4-\left(w_{0}^{2} \pi / \lambda\right)^{2}}\right|,
$$

where $R_{\mathrm{PR}}$ is the radius of curvature of mirror $\mathrm{M}_{\mathrm{PR}}$ and $w_{0}$ and $z_{0}$ are the radius and position of the waist of the incident beam, respectively. If this equation is fulfilled, the symmetric output port $(01,10)$ of a power-recycled interferometer with grating beam splitter can show perfect destructive interference independent of the splitting ratio of the beam splitter as is the case for a Michelson interferometer with a conventional beam splitter (and identical optical loss in the arms). The given description does not hold for broadband light due to the wavelength-dependent properties of the grating.

The experimental setup is shown in Fig. 3. The light source was a Nd:YAG laser at $1064 \mathrm{~nm}$ with a spectral linewidth of about $1 \mathrm{kHz} / 100 \mathrm{~ms}$, which was spatially filtered by a traveling wave mode-cleaner

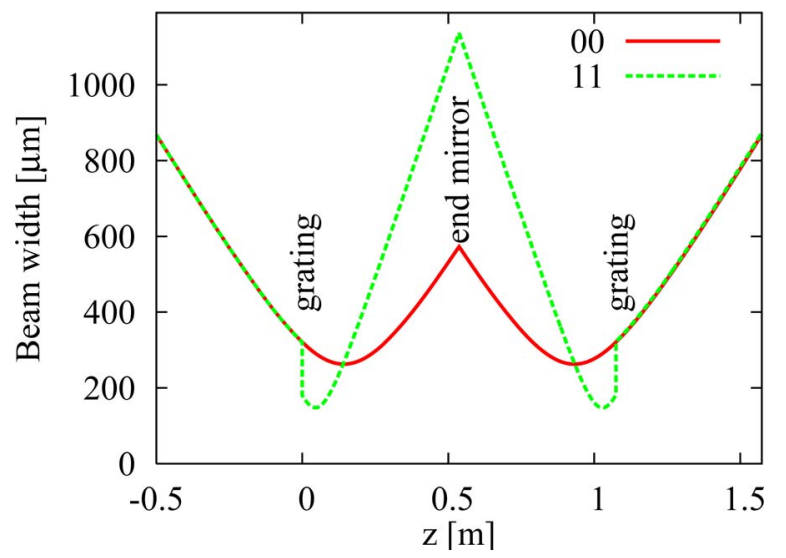

Fig. 2. (Color online) Variation of the widths of a fundamental Gaussian mode in the diffraction plane versus propagation $z$ through a Michelson interferometer with diffractive beam splitter. The saggital beam width is identical in both arms and matches to trace (00).

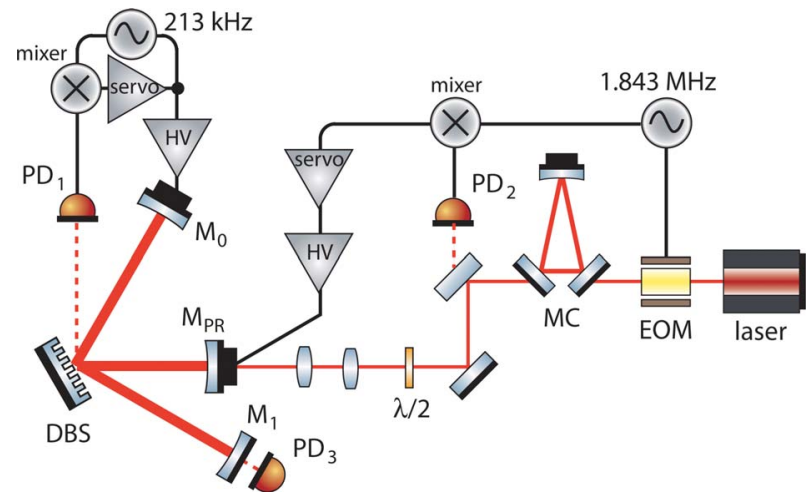

Fig. 3. (Color online) Experimental setup for the interferometric characterization of a DBS. M, mirror; MC, modecleaner; EOM, electro-optical modulator; PD, photodiode.

cavity (MC). One of the interferometer's end mirrors was mounted on a piezoelectric transducer, which was used to control the arm length and to internally phase modulate the laser beam at $213 \mathrm{kHz}$. The error signal for the electronic servo loop was derived from the optical signal in the interferometer's dark port (photodiode $\mathrm{PD}_{1}$ ). The interferometer's $\mathrm{PR}$ cavity was stabilized via the Pound-Drever-Hall (PDH) technique using the reflected light on photodiode $\mathrm{PD}_{2}$. The PDH phase modulation was achieved by means of an electro-optical modulator (EOM) at $1.843 \mathrm{MHz}$.

For a given grating with period of $d=790 \mathrm{~nm}$ and high-reflectivity end mirrors with radius of curvature $R_{0,1}=0.5 \mathrm{~m}$ we set up an experiment with $\phi_{\text {in }}=\phi_{0}$ $\approx 29^{\circ}, \phi_{1} \approx 60^{\circ}$, and an arm length of $l_{\text {arm }}=0.537 \mathrm{~m}$, which realized the geometry shown in Fig. 2. We measured an interferometer contrast (visibility) of $C=\left(I_{\max }-I_{\min }\right) /\left(I_{\max }+I_{\min }\right)=0.9989 \pm 0.0001, \quad$ where $I_{\max }$ and $I_{\min }$ denote the maximal and minimal power in the symmetric port, respectively. This value corresponds to a minimum power loss into the symmetric port (dark port) of approximately $(1-C) / 2$ $=(550 \pm 50) \mathrm{ppm}$. The position of the PR mirror with $R_{\mathrm{PR}}=-0.6 \mathrm{~m}$ was fixed according to Eq. (1). Measurements of the ratio between the power in the symmetric port $\left(\mathrm{PD}_{1}\right)$ and the power inside the $\mathrm{PR}$ cavity $\left(\mathrm{PD}_{3}\right)$ for different mirror positions corroborated the calculated prediction, as it was minimal at $l_{\mathrm{PR}}$ $=0.378 \mathrm{~m}$. The dark port power loss with an inserted PR mirror was measured by means of direct power measurements to be $A_{\mathrm{dp}}=(781 \pm 137) \mathrm{ppm}$ while the interferometer and the PR cavity were stabilized on their operation points. This is in good agreement with the aforementioned contrast measurement without the PR mirror. The slightly higher value was attributed to the residual deviation of the PR mirror from its optimum position.

The realization of a power-recycled interferometer with a high interference contrast enabled the precise characterization of the optical loss of the diffractive beam splitter. For this purpose we analyzed the finesse of the PR cavity and compared it with the finesse values of two linear reference Fabry-Perot resonators (FPRs). The latter were built using the same mirrors $M_{P R}$ and $M_{0}$ or $M_{1}$, respectively. We measured the linewidths and the free spectral ranges 


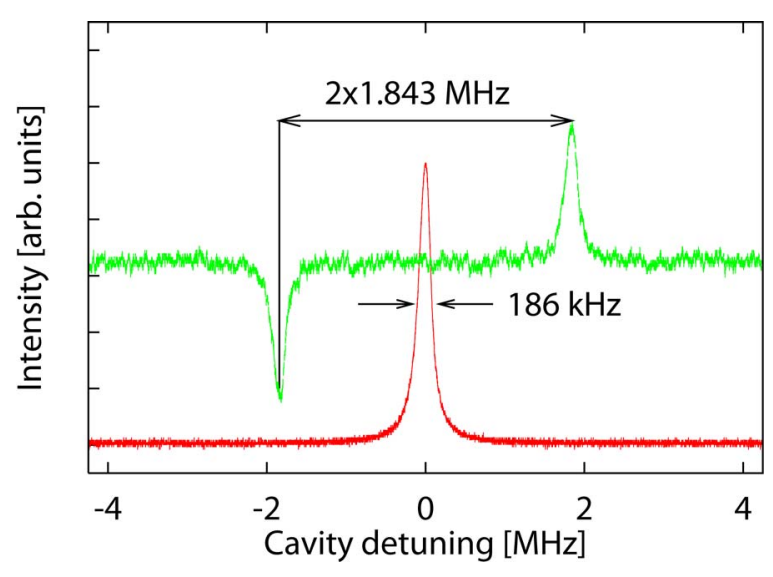

Fig. 4. (Color online) Scan over one PR-cavity resonance peak (measurement time $\approx 1 \mathrm{~ms}$ ). The tuning is calibrated with signals at $\pm 1.843 \mathrm{MHz}$.

(FSRs) of all three cavities. For the linewidth measurements we used the PDH phase modulation signals as frequency markers to calibrate the cavity length scans over their resonances. For the PR cavity we got a value of $\Delta \nu=(186 \pm 6) \mathrm{kHz}$; see Fig. 4 . The FSRs were measured by means of cavity length measurements. We got a value of $f_{\mathrm{FSR}}$ $=c / 2 /(0.915 \pm 0.002) \mathrm{m}$ for the PR cavity, with $c$ being the speed of light. The finesse is given by the fraction of those two values and was found to be $\mathcal{F}_{\mathrm{PR}}$ $=883 \pm 28$ for the PR cavity, with a marker uncertainty of $\pm 1 \%$ included. The finesse values of the reference FPRs were found to be $2297 \pm 73$ and $2820 \pm 89$. Those values resulted in a combined reference value of $\mathcal{F}_{\text {ref }}=2543 \pm 81$, taking the grating's splitting ratio $(47.8 / 52.2 \pm 0.1) \%(0 \mathrm{th} / 1 \mathrm{st})$ into account. The $\mathcal{F}_{\text {ref }}$ value is also given by

$$
\mathcal{F}_{\text {ref }}=\pi / \arccos \left[1-\left(1-\rho_{\text {PR }} \rho_{\text {end }}\right)^{2} /\left(2 \rho_{\text {PR }} \rho_{\text {end }}\right)\right],
$$

where $\rho_{\mathrm{PR}}$ and $\rho_{\text {end }}$ are the amplitude reflectivities of the coupling mirror and (the averaged) end mirror,

Table 1. Error Propagation

\begin{tabular}{ccc}
\hline Quantity & Error & $\begin{array}{c}\text { Projected Error } \\
\text { for } A(\mathrm{ppm})\end{array}$ \\
\hline PR-cavity length & $\pm 2 \mathrm{~mm}$ & \pm 15 \\
Linewidth & $\pm 3 \%$ & \pm 213 \\
Marker frequency & $\pm 1 \%$ & \pm 71 \\
$\mathcal{F}_{\text {ref }}$ & \pm 81 & \pm 78 \\
Total rms error & & \pm 238 \\
\hline
\end{tabular}

respectively. The $\mathcal{F}_{\mathrm{PR}}$ value is reduced due to an additional cavity round-trip loss $A$ that can be allocated to the amplitude reflectivity of the second mirror by a factor $\sqrt{1-A}$. Using Eq. (2) the overall additional loss was determined to $A=(0.463 \pm 0.024) \%$ with an $\mathrm{rms}$ error based on the error propagation listed in Table 1. $A$ is the sum of the measured dark port loss $A_{\mathrm{dp}}$, which includes all wavefront disturbing effects, and twice the total optical loss of the grating. The latter was finally derived to $A_{\mathrm{gr}}=\left(A-A_{\mathrm{dp}}\right) / 2$ $=(0.193 \pm 0.019) \%$. Note that this value includes absorption, scattering, and transmission of the grating, as well as power loss into unwanted diffraction orders caused by possible fabrication errors. The splitting ratio of the diffractive beam splitter quoted above was determined by a measurement of the interference contrast at the antisymmetric port of the interferometer when the PR mirror was removed, taking the contrast at the symmetric port and the difference of the end mirror reflectivities into account.

In summary, we presented a method to precisely measure the total optical loss of diffractive beam splitters with two diffraction orders independent of its splitting ratio. We showed that a dielectrically coated reflection grating can be used as the beam splitter in power-recycled Michelson interferometers with a power-recycling cavity finesse value of almost 1000. The limitation of this value was mainly given by the total optical loss of the grating and to a lesser extent by the interference contrast achieved.

This work was supported by the Deutsche Forschungsgemeinschaft within the Sonderforschungsbereich TR7.

\section{References}

1. K.-X. Sun and R. L. Byer, Opt. Lett. 23, 567 (1998).

2. A. Bunkowksi, O. Burmeister, P. Beyersdorf, K. Danzmann, R. Schnabel, T. Clausnitzer, E.-B. Kley, and A. Tünnermann, Opt. Lett. 29, 2342 (2004).

3. R. Schnabel, A. Bunkowski, O. Burmeister, and K. Danzmann, Opt. Lett. 31, 658 (2006).

4. S. Hild, H. Lück, W. Winkler, K. Strain, H. Grote, J. Smith, M. Malec, M. Hewitson, B. Willke, J. Hough, and K. Danzmann, Appl. Opt. 45, 7269 (2006).

5. W. Winkler, K. Danzmann, A. Rüdiger, and R. Schilling, Phys. Rev. A 44, 7022 (1991).

6. D. Schnier, J. Mizuno, G. Heinzel, H. Lück, A. Rüdiger, R. Schilling, M. Schrempel, W. Winkler, and K. Danzmann, Phys. Lett. A 225, 210 (1997).

7. S. Fahr, T. Clausnitzer, E.-B. Kley, A. Tünnermann, Appl. Opt. 46, 6092 (2007).

8. A. E. Siegman, J. Opt. Soc. Am. A 2, 1793 (1985). 